\title{
INFLUENCE DE LA PROXIMITÉ REPRÉSENTATIVE SUR LA MIXITÉ FEMMES-HOMMES DANS LES PETITES ENTREPRISES ARTISANALES DU BÂTIMENT
}

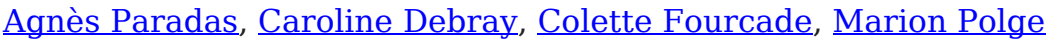

De Boeck Supérieur | « @GRH »

2019/4 nº 33 | pages 119 à 147

ISSN 2034-9130

ISBN 9782807393172

Article disponible en ligne à l'adresse :

https://www.cairn.info/revue-agrh-2019-4-page-119.htm

Distribution électronique Cairn.info pour De Boeck Supérieur.

(C) De Boeck Supérieur. Tous droits réservés pour tous pays.

La reproduction ou représentation de cet article, notamment par photocopie, n'est autorisée que dans les limites des conditions générales d'utilisation du site ou, le cas échéant, des conditions générales de la licence souscrite par votre établissement. Toute autre reproduction ou représentation, en tout ou partie, sous quelque forme et de quelque manière que ce soit, est interdite sauf accord préalable et écrit de l'éditeur, en dehors des cas prévus par la législation en vigueur en France. Il est précisé que son stockage dans une base de données est également interdit. 


\section{INFLUENCE DE LA PROXIMITÉ REPRÉSENTATIVE SUR LA MIXITÉ FEMMES-HOMMES DANS LES PETITES ENTREPRISES ARTISANALES DU BÂTIMENT}

\section{Agnès Paradas}

Maître de Conférences en sciences de gestion, habilitée à diriger des recherches

Université d'Avignon - MRM et LabEx Entreprendre Montpellier

agnes.paradas@wanadoo.fr

\section{Caroline Debray}

Maître de Conférences en sciences de gestion

Université Montpellier - MRM et LabEx Entreprendre Montpellier

caroline.debray@umontpellier.fr

\section{Colette Fourcade}

Maître de Conférences Honoraire, habilitée à diriger des recherches

Université de Montpellier - MRM

colette.fourcade@wanadoo.fr

\section{Marion Polge}

Maître de Conférences en sciences de gestion, habilitée à diriger des recherches

Université Montpellier - MRM et LabEx Entreprendre Montpellier

marion.polge@umontpellier.fr

\section{Résumé}

De nombreux métiers sont encore exercés par des populations exclusivement masculines ou féminines, alors que l'enjeu de la mixité femmes-hommes devient vital dans nos sociétés. L'artisanat du bâtiment, monde d'hommes, illustre ce fait. La demande d'évolution dans ce secteur relève d'attentes économiques et éthiques. Si toutes les entreprises sont touchées par ce phénomène, l'observation de petites structures permet d'identifier certaines caractéristiques propres à la taille réduite. En particulier, dans un champ de questionnements touchant fortement aux représentations et stéréotypes, 
la place centrale des dirigeants conduit à s'interroger sur l'influence de la proximité représentative sur la mixité. À partir d'une étude qualitative en deux temps - entretiens et focus groups - la présente recherche tente de mettre en perspective les éléments déterminants de l'intégration des femmes dans les métiers de l'artisanat du bâtiment, et de faire des propositions conduisant à l'amélioration de la mixité.

Mots-clés

Artisanat du bâtiment, représentations, stéréotypes, mixité femmes-hommes, petite entreprise, performance.

\section{INTRODUCTION}

Depuis plus de dix ans (Scotto et al., 2008), le BTP connaît des départs à la retraite massifs, et près de 30000 départs sont encore prévus dans les cinq années à venir (action BTP, 2019'). Le secteur doit relever le défi de l'attrait auprès des populations, passant par la sensibilisation des jeunes, mais également d'une population peu présente jusque-là : les femmes. L'enjeu est donc de taille puisqu'il doit conduire à intégrer des femmes dans un milieu traditionnellement très masculin. De façon concomitante, les entreprises, de toutes tailles et tous secteurs sont de plus en plus incitées à développer leur responsabilité sociétale, qui devient une condition de performance. Les petites entreprises artisanales du bâtiment, empreintes d'une forte identité, n'échappent pas à ces questions, et ce de manière peut-être plus contraignante. Au sein de la RSE, la mixité femmes-hommes, inscrite dans la diversité, tient une place importante. Dans les petites entreprises, il est souvent davantage question de l'engagement responsable du dirigeant que de responsabilité de l'entreprise. Ainsi, le dirigeant est l'acteur incontournable de tout changement, et ce sont donc ses croyances, représentations et valeurs qui vont porter les stratégies développées.

\section{Le dirigeant étant aux commandes du système de gestion de son organisation et le premier décideur en termes de choix de recrutements, il est alors plau- sible de supposer que le développement de la mixité femmes-hommes dans les entreprises artisanales du bâtiment va passer d'abord par une évolution de ses représentations.}

Les attentes de la société et les recherches sont croissantes dans le domaine de la diversité, et plus particulièrement de la mixité, au sein des entreprises. Un travail sur la mixité dans le secteur du bâtiment constitue clairement une recherche au service du

[1] http://www.actionbtp.com/chiffres-clefs.html 
terrain, permettant toutefois de faire également évoluer les concepts. Au-delà d'une exigence sociale d'intégration et de non-discrimination, les dirigeants de PME ont en effet intérêt à développer la mixité, porteuse de performance sociétale et d'entreprise, ce qui a pu être corroboré par des dirigeants et DRH d'entreprises de grande taille (Polge et al., 2017). L'intérêt d'observer des petites entreprises dans ce mouvement entre représentations et évolution des pratiques, est la proximité reconnue dans les petites structures qui permet - au dirigeant et aux observateurs - de percevoir plus directement les conséquences des actions mises en œuvre, et les effets de bouclage, vicieux ou vertueux. Ainsi, souvent, les PE sont considérées comme outils de recherche (Goy, 2006 ; Dupuis et al., 2006) car l'observation peut se faire dans de meilleures conditions et offre dans ce cadre des occasions de traduire des problématiques sociétales de manière beaucoup plus directe, vivante, et intelligible, que par référence au cadre habituel des grandes entreprises.

Les études concernant les questions de femmes se développent depuis quelques années, mais sont davantage traitées avec une approche sociologique ou macro-économique, et moins avec un regard psychosociologique autour des stéréotypes et préjugés. Les questions des femmes dirigeantes, entrepreneures, manageures, de la gestion de carrière et du plafond de verre, ou encore de la féminisation des 'Top Management Team', dominent largement (Falcoz, 2016 ; Santoni et Barth, 2014). Notre travail, à propos des stéréotypes liés à l'emploi de femmes salariées, est ainsi tout à fait pertinent, car il traite d'un sujet plutôt rare dans le domaine.

Afin de répondre au mieux à cette question du rôle majeur des stéréotypes - du dirigeant et des autres acteurs - sur le développement des pratiques de mixité dans les petites entreprises artisanales du bâtiment, une étude qualitative auprès d'artisans et de parties prenantes, intégrant entretiens et groupes de discussion (focus groups) a été menée à grande échelle dans une région, sur plusieurs départements. Dans une logique abductive, nous exposerons tout d'abord la méthodologie de l'étude qui a nourri notre réflexion, puis nous présenterons volontairement de manière conjointe, les sources théoriques et les résultats obtenus.

Ainsi, dans une première partie, le protocole d'étude et la situation de la mixité sur le terrain observé seront décrits. Les concepts utiles à la compréhension du travail, en particulier ceux de proximité représentative et de stéréotypes de genre seront explicités. Une deuxième partie permettra d'identifier les principaux enjeux, en particulier par le biais de l'observation des freins majeurs à la mixité et de la relation entre la mixité et la performance. Enfin, une troisième partie s'attachera à la prescription et aux sources de progrès, en faisant d'abord un focus sur l'observation des femmes dirigeantes, signe fort de l'influence des représentations des dirigeants sur la mixité, pour ensuite proposer quelques pistes d'action. 


\section{SPÉCIFICITÉS DU TERRAIN ET IMPACT SUR LES REPRÉSENTATIONS ET LA MISE EN FUVRE DE LA MIXITÉ}

Dans un premier temps, il semble donc essentiel de se focaliser sur la méthodologie de l'étude ayant permis la réflexion autour de l'importance des représentations et stéréotypes dans le cadre de l'artisanat du bâtiment.

\section{, 1.1 L'approche du terrain : méthodologie et état de la mixité dans le secteur}

L'étude réalisée va bien au-delà de ce qui sera présenté ici, seule une partie des investigations ayant été prise en compte. Elle s'insère dans un secteur qui présente des spécificités de genre qu'il faudra préciser.

\subsubsection{Des données issues d'une étude qualitative multiple}

Notre présentation émerge d'une recherche qui a été réalisée sur une durée de deux ans. L'Université de Montpellier a initié une démarche pilote sur la mixité et l'égalité femmes-hommes dans le secteur de l'artisanat du bâtiment sur les départements de la Région Occitanie. L'objectif affiché était de sensibiliser à la mixité femmes-hommes, par la mise en place d'outils d'accompagnement co-construits avec les acteurs du secteur et les chercheurs de I'Université. Les enjeux généraux se sont focalisés sur l'obligation juridique, mais également sur la performance de l'entreprise et sur le caractère stratégique d'un meilleur équilibre des équipes en termes de sexe des collaborateurs. Répondant à la double injonction économique et sociale, ce projet s'est inscrit dans une approche socialement responsable.

L'objectif final était d'améliorer la mixité femmes-hommes dans les entreprises. Pour cela plusieurs missions ont été menées : faire un état des lieux de la situation et de la place des femmes dans les entreprises artisanales du bâtiment en région Occitanie, identifier les outils existants et à créer, mettre en place un protocole de recherche auprès d'un large panel de parties prenantes, co-construire des actions collectives au sein d'un dispositif RSE et créer un observatoire en matière de mixité femmes-hommes dans le secteur.

Un protocole de recherche large a été déployé pour répondre à ces questions et se décompose de la façon suivante : une recherche documentaire, des études qualitatives de deux types dans 4 départements auprès des artisans et des parties prenantes dans le secteur de l'artisanat du bâtiment. Pour cela, 22 entretiens individuels et 10 entretiens de groupes (focus groups) ont été réalisés. Enfin, une enquête quantitative a également été menée.

Cet article se focalisera sur les résultats des études qualitatives, aux résultats déjà très fournis. Seules les données liées à notre problématique seront utilisées. 
Concernant les entretiens, 13 entretiens ont été menés auprès d'artisans et 9 auprès de parties prenantes (Annexe 1). Ils ont été menés selon un guide d'entretien structuré autour de 5 items : profil de l'enquêté, stéréotypes, freins/motivations, performance, connaissance de la RSE. Le déroulé des entretiens s'est étendu sur une heure trente en moyenne, et a fait l'objet d'enregistrements. L'exploitation des données a été réalisée selon 3 étapes : retranscription des verbatim, analyse textuelle à partir des verbatim, établissements de tableaux d'exploitation comparatifs.

Les 10 focus groups (FG) ont permis de réunir entre 6 et 12 artisans ou parties prenantes, volontaires pour venir discuter ouvertement dans une logique de créativité sur la question de la mixité femmes/hommes dans l'artisanat du bâtiment (Annexe 2). II a ainsi été nécessaire de rechercher un panel de profils de professionnels le plus représentatif possible du secteur. Parmi les parties prenantes, la démarche a été de convier aux FG les parties prenantes primaires (en relation contractuelle directe et essentielle avec l'entreprise : clients, fournisseurs, salariés, prestataires : avocats, comptables...) et secondaires (acteurs diffus dans l'environnement, partenaires institutionnels, fédérations professionnelles, élus locaux, milieu associatif). Ainsi, la technique des focus groups s'ancre dans le terrain et a pour objectif de répondre aux " pourquoi » et " comment ". Ce type d'animation qualitative, à la fois orale et en groupe vise le recueil de perceptions, des attitudes et des croyances, mais également cherche à relever les freins et zones de résistance des groupes ciblés, le principe étant celui de la discussion libre autour des thèmes proposés. Le diaporama support était commun à tous les focus groups, les séances enregistrées, et il était indispensable que toutes les séances utilisent les mêmes outils et méthodes. L'une des originalités de la méthode est d'utiliser des mots-clefs ainsi que des phrases "chocs " extraites d'entretiens individuels préliminaires avec des artisans, afin de "libérer » la parole des acteurs. L'animateur reformule, précise et relance les discussions et fait une synthèse de chaque thème évoqué, synthèse qui doit être soumise pour approbation aux parties prenantes invitées, de manière à s'assurer de la validité des résultats et des interprétations, et à assurer une amélioration continue des processus. Au final, 6 catégories ont été retenues pour le traitement : la présence des femmes dans l'artisanat du bâtiment ; la représentation de la mixité à travers les stéréotypes ; la complémentarité des rôles et des missions ; les thèmes de mixité, efficacité, efficience, effectivité (composantes de la performance) ; la mixité dans la formation et le recrutement; et enfin, quel avenir pour la mixité ?: idées et propositions.

Des résultats très proches ont été obtenus lors des entretiens et des focus groups concernant les conclusions sur les représentations et stéréotypes. Les seules différences notables constatées sont liées à la conscience de l'existence de ces stéréotypes, plus forte pour certaines parties prenantes déjà engagées dans l'analyse de la situation de mixité. II est donc possible de relier les données obtenues lors des entretiens et des focus groups. 


\subsubsection{La mixité dans le secteur de l'artisanat du bâtiment}

Sur les 87 familles professionnelles recensées, seules 13 sont mixtes (CESE, 2014) soit $17 \%$ des métiers représentant $16 \%$ des emplois. Parmi les facteurs associés au fait qu'une femme réalise un métier encore très majoritairement exercé par les hommes, entrent en ligne de compte l'origine géographique et sociale et la situation familiale. Selon Klinkenberg (2016), " on constate que le taux de chômage des filles sortant des filières dites masculines, quel que soit leur niveau de qualification, est plus élevé que celui des garçons issus de ces mêmes sections ". La situation est cependant différente selon les métiers et niveaux de qualification, notamment parce que l'influence des stéréotypes de genre peut varier selon les milieux sociaux.

Le secteur du bâtiment est très cosmopolite, diversifié en termes d'âge, de nationalités, de cultures et de religion... mais pas de genre. L'artisanat du bâtiment n'est donc pas un cas isolé. En 2015, on comptabilisait 11,9 \% de femmes dans le bâtiment dont 46,1 \% parmi les employés et techniciens, $18 \%$ parmi les cadres et 1,5\% parmi les ouvriers. Naves et Wisnia-Weill (2014) indiquent d'ailleurs qu'à caractéristiques comparables (âge, niveau et spécialité de diplôme, localisation géographique, origines géographiques et sociales), les femmes seules exercent davantage une profession d'ouvrier qualifié de la sphère artisanale à dominante très masculine, les femmes en couple avec au moins un enfant de moins de 18 ans étant en revanche sous-représentées.

L'étude a confirmé le fait que les statuts, postes ou métiers occupés majoritairement par des femmes concernaient principalement la conjointe, les postes administratifs, les postes de conception, voire de direction d'équipe davantage que les postes d'exécution, et enfin les métiers à composante esthétique au détriment des métiers plus pénibles. L'identité masculine étant particulièrement forte dans ce secteur, des peurs liées à la différence de sexe se sont exprimées. Enfin, il semblerait que les femmes déjà présentes le soient soit par nécessité de reconversion, soit par passion lorsqu'il s'agit d'un choix (Carlier et Marlier, 2016).

\section{, 1.2 Représentations et stéréotypes de genre}

Les notions générales de représentations et stéréotypes sont empruntées à des disciplines autres que les sciences de gestion. II s'agira d'en préciser rapidement les contours et d'en situer l'importance dans une petite entreprise. Les stéréotypes liés au genre seront ensuite étudiés.

\subsubsection{La notion de représentation au cœur des petites entreprises : partir de la proximité représentative}

La notion de représentations sociales se situe à l'interface du psychologique et du social, de l'individu et du collectif. Ce sont des manières de penser, de s'approprier, d'interpréter sa réalité quotidienne et son rapport au monde et qui sont liées aux croyances, 
valeurs et cadre de référence. La croyance, consciente ou inconsciente, serait alors un état mental consistant à considérer qu'une certaine représentation est vraie. Les valeurs, enfin, peuvent être identifiées comme des croyances fondamentales ayant trait à la façon dont on doit agir afin d'atteindre les buts que l'on juge importants (Moscovici, 1961 ; Jodelet, 2003 ; Abric, 2011).

Les stéréotypes sont des croyances partagées concernant les caractéristiques personnelles, généralement des traits de personnalité, mais souvent aussi des comportements (Leyens et al., 1996). Ills semblent donc en relation directe avec les représentations. Les stéréotypes (plutôt d'ordre cognitif) et les préjugés (relevant plutôt d'attitudes liées à notre part émotionnelle) font partie de notre héritage culturel. Nous intégrons des normes et des valeurs tout au long de notre vie, par le biais de nos perceptions et de notre cerveau qui traite les informations qu'il reçoit de l'environnement.

Il est important d'identifier le fait que les stéréotypes peuvent exister sous différentes formes.

Les stéréotypes « directs " sont liés à ce que l'on croit à propos de l'autre. Les stéréotypes croisés vont renvoyer à ce que l'on pense que l'autre pense, c'est-à-dire la représentation que I'on a des représentations d'autrui (" je suis dans un milieu d'hommes, on attend de moi que je me comporte comme un homme »). Enfin, les auto-stéréotypes concernent la façon dont on se représente nos propres stéréotypes (" je pense qu'il n'y a pas de différence entre les hommes et les femmes... mais dans les milieux de femmes, on voit bien que ça chipote pour rien "). Un stéréotype n'est bien sûr pas forcément négatif.

Les entreprises de l'artisanat du bâtiment tirent leur spécificité de leur activité mais aussi du fait que ce sont des structures de petite taille. Leur faible dimension induit un certain nombre de conséquences quant à l'importance des représentations et stéréotypes. Absence de formalisation et centralisation sont deux caractéristiques qui vont induire des comportements particuliers.

Les stratégies et politiques étant peu formalisées, la socialisation et l'intégration des normes informelles, qui peuvent être acquises grâce à la qualité des relations, est une dimension qui contribue au pilotage implicite de l'entreprise. Dans les PE, chaque salarié est ainsi un maillon essentiel de la chaîne, et l'imbrication de chaque maillon est déterminant de la performance. Selon Mahé de Boislandelle (1996), des effets de grossissement influencent l'importance des relations interpersonnelles. Chaque personne dans l'entreprise a une place relativement plus importante, ce qui va induire une forte interdépendance dans les attitudes et comportements de chacun. De la même manière, les parties prenantes seront considérées comme appartenant au groupe de proximité, et donc les interactions personnelles bien plus fortes que dans des structures plus grandes. La place prépondérante du dirigeant et la forte centralisation présente dans les petites entreprises exercent certainement un rôle majeur dans la diffusion des valeurs, croyances, représentations et stéréotypes dans le groupe et dans le système de gestion. 
Les dirigeants, de par leur vision et leurs représentations personnelles, professionnelles et sociales (Paradas, 2007) vont définir des stratégies adaptées à leurs attentes et leurs conceptions. De plus, à la différence des plus grandes structures, le dirigeant est souvent le propriétaire, et ses choix lui appartiennent, non dictés par une gouvernance supérieure qui aurait d'autres motivations que lui.

À partir des travaux de Torres (2004) qui pointent l'égotrophie du dirigeant et déclinent la proximité dans les petites entreprises sous des formes variées [hiérarchique (peu d'intermédiaires), fonctionnelle (peu de spécialistes), temporelle (réactivité, souplesse), de l'information, des relations commerciales, fusion patrimoine dirigeant et entreprise], Paradas (2008) ajoute le concept de proximité représentative. Considérant que le dirigeant fait preuve d'un nombrilisme qui le pousse à tout rapporter à sa personne, les enjeux stratégiques vont être souvent réfléchis en termes psychosociologiques. Le dirigeant va ainsi orienter son système de gestion et son système relationnel en fonction de sa vision du monde et de ses représentations.

Selon les observations, les dirigeants d'entreprise engagés dans une réelle démarche de mixité sont convaincus du bien-fondé et des plus-values apportées par celle-ci. Sans cette conviction, il semble difficile de parvenir à l'amélioration des pratiques. Concernant le dirigeant lui-même, sa sensibilité, sa personnalité, peuvent être à l'origine d'une politique plus ou moins volontariste en matière de mixité.

Ainsi, la diversité dans les petites organisations peut être observée à l'aune de ces considérations. Dans le cadre de la mixité, l'idée selon laquelle une femme peut être aussi efficace et efficiente qu'un homme relève fortement des stéréotypes et des représentations. Si la mixité femmes-hommes semble bien admise dans certaines branches, elle peut poser des difficultés dans des secteurs traditionnellement très masculins (le bâtiment) ou très féminins (les administrations ou les services à la personne). Ce ne serait ainsi pas le fait d'être un homme ou une femme qui poserait problème, mais plutôt celui d'arriver en tant que sexe opposé dans un groupe. Louey et Schütz (2014), citant les travaux précurseurs de Williams en 1992, montrent que les hommes qui exercent des activités pensées comme féminines bénéficieraient d'avantages structurels : moins de freins à l'embauche, un meilleur accueil de l'équipe féminine en place et une évolution de carrière vers les tâches mieux valorisées de l'activité ... Les difficultés semblent ainsi plus souvent concerner le cas de l'arrivée d'une femme dans un groupe masculin ... Notre société se trouve encore dominée par des représentations culturelles qui entravent dans une large mesure la participation active, responsable et autonome de l'élément féminin. Le système culturel résiste encore à un accès ouvert du féminin aux sphères et tâches prestigieuses de la vie sociale - ces dernières étant souvent réservées au masculin.

\subsubsection{Les représentations et stéréotypes de genre}

La Direction de l'Égalité des Chances de la Communauté Française définit le stéréotype de genre comme « toute représentation (langage, attitude ou représentation) péjorative 
ou partiale de l'un ou l'autre sexe, tendant à associer des rôles, comportements, caractéristiques, attributs ou produits réducteurs et particuliers à des personnes en fonction de leur sexe, sans égard à leur individualité. II déclenche une double démarche par laquelle on réduit l'identité femme et l'identité homme à un certain nombre de rôles, de comportements, de caractéristiques et d'attributs tout en imposant aux individus d'être un homme ou une femme en cadrant à ces seuls rôles, comportements, caractéristiques, etc. ".

Concernant les définitions, le sexe renvoie à une réalité physique, alors que le genre permet d'identifier un phénomène de représentation (comment on se perçoit et comment on se comporte). Le sexe fait référence à la nature et le genre à la culture et à une construction sociale (Tostain, 2016). Selon Klinkenberg (2016), le sujet est d'autant plus délicat que " le concept de genre, bien instrumentalisé dans tous les débats de société, est largement méconnu, tant du grand public que chez les décideurs ". Elle ajoute que " les questions où le sexe est impliqué sont, plus que d'autres, génératrices de peurs et de fantasmes, sur lesquels on imagine qu'un psychanalyste aurait des choses à dire " et plus loin, que " les réflexions et les recherches sur le genre se sont aussi faites plus sociopolitiques".

Les stéréotypes et préjugés font partie de notre héritage culturel. Ils sont souvent masqués par des démonstrations se voulant factuelles, comme le rôle des hormones ou la maternité (Zuinen, 2002) ou plus sociales comme par exemple la position de maîtresse de maison ou la qualité relationnelle d'attention à l'autre (Klinkenberg, 2016 ; Lebègue, 2015 ; Vier Machado et Rouleau, 2002 ). Les arguments en faveur des différences ne manquent pas. Chicha (2013) identifie un certain nombre de stéréotypes et d'idées préconçues au sujet des qualités et des défauts présumés des femmes qui, à son avis, expliquent en large partie la ségrégation observée. Les négatifs renvoient à l'absence de force physique, la fragilité et le manque de résistance à un environnement pénible (danger, chaleur, etc.), le manque d'expérience professionnelle, de motivation, de compétence, de confiance en soi, de disponibilité, les dangers de la mixité, le danger du travail de nuit, la réticence des collègues ou le refus des clients. Les positifs concentrent les femmes dans certaines professions où sont requises certaines qualités : il peut s'agir de la minutie, de la dextérité ou de la motricité fine, de la concentration et la patience, la loyauté et la docilité ou les moindres besoins financiers. Les raisons exprimées par les interlocuteurs, dirigeants, cadres ou employés paraissent aller de soi. Selon Cornet et Bonnivert (2008), des qualités " féminines » de bienveillance, d'attention à l'autre, orientées sur le relationnel et centrées sur la motivation des subordonnés seraient toutefois gage de performance pour l'entreprise car synchrones avec les évolutions profondes de l'entreprise et de la société. Plusieurs auteurs cités par Vier Machado et Rouleau (2002), précisent également que la gestion féminine serait caractérisée par une pratique du pouvoir plus horizontale et participative, par une recherche de satisfaction des proches et des employés et par un intérêt pour la responsabilité sociale. L'exercice du leadership 
serait plus démocratique et relationnel et les entrepreneures porteraient une attention particulière à l'équité et au bien-être de la communauté (Santoni et Barth, 2014).

Toutefois, à se focaliser sur les "différences » (approche inhérente à une lecture en termes de diversité), le risque est réel de valoriser des qualités soi-disant féminines (précision, patience, dévouement) ou un management soi-disant féminin (écoute, empathie, consensus) tout en plaçant ces qualités plus bas dans la hiérarchie des besoins de l'entreprise (Laufer, 2003, Klinkenberg, 2016).

Pourtant, d'autres études viennent infirmer les croyances profondes. Catherine Vidal (2006) montre dans ses recherches sur le cerveau que sur 1000 études, seulement $3 \%$ ont indiqué une différence entre les sexes. Nous aurions les mêmes dispositions biologiques et neurologiques à la naissance que nous soyons homme ou femme. Et d'après I'auteure, l'extension du cortex permet chez l'être humain d'échapper au déterminisme des gènes et des hormones. Cela mettrait au centre des réflexions l'importance des facteurs individuels, sociaux et culturels (Marsan, 2007). L'enfant développerait d'ailleurs son identité de genre seulement entre 24 et 36 mois (Klinkenberg, 2016).

Et lorsque les comportements gestionnaires sont observés, comme souligné dans la méta-analyse de Sarah Saint-Michel (2010), il semble possible d'affirmer l'inexistence de différences de compétences de leadership entre hommes et femmes. Finalement, les construits sociaux autour des différences sexuées sont bien présents (Dubar, 1998) doublés de stéréotypes qui ont la vie dure.

La société étant en dynamique constante, on observe toutefois que la tendance serait à l'évolution des représentations autour du féminin. Plusieurs philosophes et sociologues (Marsan, 2007) perçoivent un retour au féminin, ainsi qu'une orientation plus féminine des valeurs de la société (Luyckx, 2002). Ce dernier cite deux recherches, l'une aux États-Unis et l'autre en Europe, montrant une évolution du système de valeurs implicites des citoyens vers des valeurs plus féminines. Le paradigme patriarcal serait ainsi en train d'évoluer vers une société où « les visions et intuitions des femmes seraient prises en compte ». En parallèle, on peut se demander dans quelle mesure l'influence des valeurs féminines dans la société amène à une nouvelle façon de voir le monde et d'agir sur lui, par exemple dans une recherche de coopération plus que de compétition, ou bien si, au contraire, c'est l'émergence inéluctable des responsabilités (climat, société ...) qui induit des préoccupations d'ordre plus féminin. La féminisation des valeurs de la société traduit une progression, chez les hommes comme chez les femmes, de l'importance attachée à l'esthétique, aux émotions, à la coopération entre individus et à la prise en compte du long terme, cela expliquant en partie le développement des réseaux sociaux et le souci croissant de développement durable, comme le confirment les résultats de l'enquête de Saint-Pierre et al. (2011).

À partir d'une étude concernant les femmes dans le bâtiment et de questions autour de l'image et des représentations sociales dans la Construction, Scotto et al. (2008) 
reprennent certaines de ces considérations générales. L'intégration " limitée " est évidemment constatée. Les stéréotypes sont déterminants et variés. Les femmes qui sont présentes dans le secteur montreraient une force de caractère qui les pousserait à refuser les schémas traditionnels. Les qualités dites féminines utiles à l'exercice du métier sont avancées, comme la méthode, le soin ou l'esthétisme, associées à une intelligence d'organisation des modalités du travail.

II est possible de retrouver certains de ces stéréotypes personnels au travers de l'étude menée. Les hommes interrogés identifient chez les femmes les qualités suivantes : soin, rigueur, intégrité, meilleure acquisition, communication, approche consciencieuse ; et des défauts liés à l'absence de force, le côté pointilleux, l'absence de compromis ou le besoin de reconnaissance. Les femmes, elles, se jugent comme professionnelles et plus compétentes en relations humaines. Elles s'associent à l'idée qu'elles auraient une force insuffisante et certaines, par conviction ou par résignation?, avancent l'idée qu'elles ne sont pas là pour décider mais pour accompagner.

Ces considérations générales étant posées, il semble important de s'interroger sur la puissance des stéréotypes en présence et en particulier d'identifier l'impact des représentations sur le fonctionnement de l'entreprise.

Dans le développement qui va suivre, le choix a été fait d'associer aux résultats de recherches d'autres auteurs, des descriptions issues des données recueillies dans notre étude.

\section{DES REPRÉSENTATIONS TRÈS RICHES AUTOUR DE LA PERFORMANCE}

La question de la performance, dans son acception la plus large, est centrale dans les organisations. Les perspectives plus pessimistes insistent sur l'impact négatif de la diversité sur la performance des groupes de travail (développement de tendances communautaristes) alors que les plus optimistes pensent la diversité comme jouant un rôle positif sur la performance économique de l'entreprise (Belghiti-Mahut et al., 2014 ; Belghiti et Rodhain, 2001 ; Bruna et Chauvet, 2010 ; Landrieux-Kartochian, 2005). Les représentations sexuées sont bien sûr associées à cette question. II semble ainsi pertinent d'observer les freins à la mixité avant d'en identifier les sources de performance.

\section{, 2.1 Les freins à la mixité}

Les éléments factuels avancés quant à la difficulté d'intégrer des femmes dans les équipes peuvent être recevables mais masquent souvent des stéréotypes sous-jacents. Le rôle des représentations sociales dans la compréhension de ces blocages est à considérer. Par exemple, s'il semble que la force physique ou les conditions de pénibilité (Gallioz, 2006 ; CNISF, 2014) soient spontanément citées comme blocages à la mixité, il 
apparaît que la force de la tradition, de la culture et des représentations soit également très prégnante. Toutes les questions liées aux différences sexuées, aux phénomènes de socialisation, à l'identité ou aux blocages cognitifs pourront être explorées pour mieux comprendre les freins à la mixité (Cornet et Constantinidis, 2004 ; Sénac, 2015). Les stéréotypes sexués concernent tout aussi bien un sexe que l'autre. La problématique du métier renvoie donc à des questions de représentation par le genre et de la façon dont les individus associent métier et degré de masculinité ou de féminité (Gavoille et al., 2014).

Les stéréotypes et les préjugés entretenus à l'égard des femmes sont à la source de multiples discriminations directes. Chicha (2013) propose plusieurs illustrations de ces discriminations: le fait d'exclure les femmes des postes de direction, sous prétexte qu'elles ne savent pas faire preuve de leadership, refuser d'office de les embaucher dans certaines opérations de fabrication en invoquant leur manque de force physique, ou refuser d'embaucher une femme comme avocate ou conseillère financière parce que les clients ne voudront pas transiger avec elle. Enfin l'auteure évoque également les discriminations indirectes en citant des pratiques conçues sur le modèle du travailleur masculin dont les obligations familiales sont soit minimes, soit prises en charge par la conjointe (horaires décalés, heures supplémentaires). Enfin, les réseaux de connaissances des employés jouent un rôle majeur dans l'embauche et si les employés en place sont masculins, il est fort probable que leur réseau professionnel le soit aussi, ce qui aura tendance à perpétuer l'exclusion des femmes.

Ainsi, notre société se trouve encore dominée par des représentations culturelles qui entravent dans une large mesure la participation active, responsable et autonome de l'élément féminin. Différents arguments sont avancés.

\subsubsection{Les freins de nature physique}

"Il y a quand même beaucoup de manutention, déjà pour moi c'est un frein pour qu'une femme fasse ce métier ". Ce verbatim résume la pensée de plusieurs parties prenantes et artisans. II a déjà été question de la pénibilité au travail, particulièrement pertinente dans le bâtiment.

Selon Carlier et Marlier (2016), " les raisons de l'exclusion des femmes du monde du travail productif relèvent parfois d'un sexisme dit " paternaliste » de la part des hommes, mais pas seulement, qui estiment devoir protéger les femmes des travaux lourds. II était habituel de considérer que les femmes étaient de fragiles créatures... "

Les femmes ne seraient ainsi pas assez fortes pour exercer la plupart des tâches (Scotto et al., 2008) et les discriminations seraient d'ailleurs justifiées par ces difficultés (Cousin, 2007 ; Daune-Richard, 1998). Toutefois, les évolutions techniques permettent de réduire la charge physique, facilitant l'insertion des femmes, mais allégeant également le travail des hommes (Kminkenberg, 2016). 
De plus, selon plusieurs témoignages, les équipements sont souvent historiquement conçus pour des équipes exclusivement masculines et ajouter des sanitaires, des sas de sécurité ou tout autre investissement exigé par les différences de sexe et souvent encadré par la législation, apparaît comme un coût supplémentaire que les dirigeants ne sont pas prêts à engager. Carlier et Marlier (2016) évoquent également cet " argument de poids", tout en indiquant que " prévoir, dans le cadre des marchés publics, le redoublement automatique des locaux sociaux permettrait de neutraliser cet effet « de genre " et de raser cet obstacle à la mixité des chantiers ".

Enfin, la méconnaissance des métiers renforce ce frein, de par l'image des métiers du secteur qui est véhiculée et qui reste liée à la pénibilité et à la saleté (Carlier et Marlier, 2016).

\subsubsection{Les freins à dimension psychologique}

Le sexe dominant dans le poste apparaît comme marqueur dominant de l'emploi (Cournut-Janin, 2000). La forte identité masculine qui règne dans le bâtiment peut par exemple conduire à des prises de risque volontaires, prouvant la force du sexe masculin. On associe souvent le risque, la pénibilité, la technicité et l'outil à la masculinité, les métiers du bâtiment étant alors réservés aux garçons (Scotto et al., 2008 ; Carlier et Marlier, 2016).

Dans ce monde d'hommes, un danger guette à l'arrivée de femmes : la séduction. Elle apparaît comme un facteur potentiel de désorganisation des équipes, ou de méfiance de la femme du dirigeant, alors qu'elle est perçue de façon plus positive dans le cas d'hommes intégrant une équipe féminine (Louey et Schütz, 2014). Ces évocations reviennent régulièrement, un dirigeant racontant même la perte de l'un de ses meilleurs éléments, que sa femme ne voulait plus voir travailler dans une entreprise où il avait eu une aventure avec une femme embauchée. Sans aller jusque-là, Scotto et al. (2008) identifient également l'arrivée de femmes comme éléments perturbateurs qui bouleversent l'ambiance.

Ces difficultés créent des expériences négatives qui renforcent les idées préconçues. Elles ne facilitent pas l'intégration. D'autant que les représentations touchent non seulement les dirigeants, mais également les pairs, qui s'inquiètent d'un traitement différencié d'adaptation du métier quelquefois nécessaire. Quand ils ne sont pas indignés par le fait de devoir être dirigés par une femme ... Et finalement, disent les dirigeants, pourquoi aurait-on besoin de femmes?

La rareté des candidates vient s'ajouter aux blocages. Au-delà de l'image générale plutôt négative du métier, la situation est aggravée par les conditions de formation. L'étude a démontré très nettement l'atmosphère très négative dans les formations initiales: violence, absentéisme, et nombre de jeunes arrivés là " par défaut ». Des jeunes filles arrivant dans ce milieu, même motivées, vont souvent en sortir dégoûtées. 
II a été constaté enfin que les freins peuvent venir des artisans eux-mêmes dissuadant leurs filles: "dès le début, mon père ma dit que peintre n'était pas un métier pour les femmes", ou des conditions de vie souvent assez contraignantes, qui iraient jusqu'à tuer le couple. Certains artisans vont jusqu'à refuser de prendre des jeunes filles en stage (par exemple, le stage de découverte en classe de $3^{\mathrm{e}}$ ) par crainte de devoir gérer une mineure au sein de groupes exclusivement masculins.

Toutes ces dimensions conduisent à un certain isolement, qui renforce les difficultés déjà constatées des femmes à trouver une légitimité pour s'imposer.

Malgré tous ces obstacles, si la mixité est désirée par la société, les organisations professionnelles et les acteurs, c'est certainement parce qu'elle est porteuse de sources de performance dans l'entreprise. Si certains dirigeants ont déjà pris conscience de ces enjeux positifs, d'autres restent inscrits dans un schéma traditionnel qui bloque les évolutions. Selon Scotto et al. (2008), l'intégration des femmes au sein de ces métiers doit permettre une amélioration des comportements et des conditions de travail, profitant aux salariés dans leur ensemble. L'effet positif de surprise et d'image est également souligné. II est donc essentiel d'identifier et de communiquer ces effets positifs pour faire évoluer les représentations des plus récalcitrants.

\section{, 2.2 La mixité : source de performance?}

De manière générale, la diversité devrait être promue pour quatre raisons majeures (Cornet et Warland, 2008) : une meilleure efficacité sous I'angle productif, commercial et médiatique, la recherche d'une plus grande efficience en améliorant la mobilisation et l'usage des ressources et des compétences internes et externes à l'entreprise, le respect des lois et des règlements nationaux et communautaires et la volonté de parfaire sa réputation. Ces affirmations sont renforcées par la conviction selon laquelle la performance sociale permet la performance économique (Gauzente, 2002). Plus précisément, de nombreux auteurs affirment que la mixité de genre joue de manière significative sur la performance des organisations, et notamment sur la créativité des équipes-projets (Achin et al., 2005 ; Belghiti-Mahut et al., 2014 ; Bender et Pigeyre, 2003 ; Bruna et Chauvet, 2010 ; Catalyst, 2004 ; Landrieux-Kartochian, 2005). Les constats qui ont été faits dans l'étude appuient ces affirmations.

L'analyse des résultats nous a permis de distinguer quatre formes de performance susceptibles d'être impactées par la mixité : individuelle, organisationnelle, économique et sociale.

\subsubsection{Mixité et performance individuelle}

À écouter les acteurs concernés, la présence de femmes sur des chantiers aurait pour résultat de " tirer vers le haut » les performances individuelles des collègues hommes. Même si un lien avec des stéréotypes peut être fait, le soulignement de comportements 
professionnels différents met l'accent sur les avantages à intégrer des femmes: "le travail sera bien fait... pour les finitions, elles sont quand même meilleures "... "Une fille dans une entreprise, la mixité amène une réflexion globale sur l'assistance au chantier, et donc la sécurité ".

La relation à la clientèle plus attentive de la part des femmes amènerait ainsi les artisans hommes à soigner l'aspect commercial de leurs activités. Par contagion ou par le jeu des stéréotypes croisés, les performances individuelles des femmes dans le métier contribuent à améliorer les performances individuelles professionnelles de leurs collègues masculins. Et l'amélioration de la relation clientèle est notable, d'autant que les clients sont aussi aujourd'hui des clientes.

Bender et Pigeyre (2003), Cornet et Constantinidis (2004) ou Lambert et RamboarisonLalao (2008), font ce même constat que le recrutement de femmes peut faciliter le dialogue avec certains groupes, optimiser certaines négociations auprès de la gent féminine ou modifier le type de relationnel.

Ajoutons enfin que les dirigeants peuvent tout simplement avoir envie, par conviction, de travailler dans des équipes où la discrimination n'a pas lieu d'être. La recherche de justice peut intervenir naturellement comme facteur d'éthique personnelle du dirigeant.

\subsubsection{Mixité et performance organisationnelle}

"Moi, j'aime la mixité : j'aime parce que du coup on est meilleurs tous ensemble», dit un dirigeant interrogé. Le ressenti des dirigeants interviewés est que la mixité améliore la performance de l'organisation, à la fois intra entreprise et interentreprises. Vision différente du travail, rigueur reconnue aux femmes, en particulier pour le rangement des chantiers, apports d'idées neuves, différents éléments conduisent à une amélioration des conditions de travail et du rendu. II semblerait également que la présence mixte apaise les conflits, moins violents en présence de femmes. Les parties prenantes concernées constatent aussi une amélioration des comportements en formation, avec la présence de la mixité, notamment dans les formations professionnalisantes de jeunes actifs, des hommes affirmant qu' " une femme rend le groupe moins dissipé " Il est également apparu que les femmes déjà présentes doivent être bien motivées pour être là. Cette motivation forte peut être renforcée par l'attention des dirigeants qui souhaitent intégrer la population féminine au même titre que la masculine, et montrent une image d'entreprise soucieuse de l'équité, ce qui est très motivant (Igalens et al., 2011).

\subsubsection{Mixité et performance économique}

Les éléments précédemment cités constituent déjà des sources de performance économique. La poursuite de l'excellence, souvent évoquée par les femmes interrogées, vise en premier lieu la satisfaction de la clientèle. 
Si l'aspect financier n'est pas évoqué directement dans les réponses des personnes rencontrées, il apparaît clairement que la présence de femmes aboutit à une meilleure efficience et une amélioration de l'efficacité. La recherche d'intégration de femmes dans le secteur renvoie à une motivation importante qui est la réponse à une pénurie de compétences. Si des savoirs et savoir-faire existent sur le marché, les petites structures ont tout intérêt à en profiter (Bender, 2004 ; Meynaud et al., 2009). Des témoignages vont dans ce sens, indiquant le passage à l'embauche d'une femme par l'absence de compétences masculines disponibles. Les études du secteur BTP alertent d'ailleurs sur la nécessité de rendre le métier attrayant auprès des femmes et des jeunes pour répondre à la pénurie (Scotto et al., 2008).

La recherche de réputation et de légitimité est également évoquée. Une entreprise qui favorise la mixité apparaît comme plus moderne. Et cela peut avoir un impact en termes de parts de marché (Cornet et Constantinidis, 2004).

Enfin, le simple respect de la législation constitue une source de performance évidente, car il permet aux artisans de faire l'économie de la sanction éventuelle (Falcoz, 2016). La loi française, fondée sur la Déclaration des Droits de l'Homme, interdit toute discrimination; mais il s'agit de s'assurer de l'application de ce principe dans la sphère économique et productive (Pierre et al., 2008). La recherche de certaines certifications pourrait également être évoquée. Elle n'a cependant pas été identifiée dans notre étude.

\subsubsection{Mixité et performance sociale}

Deux niveaux de performance sociale, en relation avec la mixité, peuvent être proposés. Le premier niveau est celui d'une fluidification des relations professionnelles. Sur les chantiers, "quand il y a une femme, ça va mieux, c'est plus calme, l'atmosphère change, c'est pas moi qui le dit, ce sont les hommes", dit une femme. Notons que les dirigeants, hommes ou femmes, de petites entreprises citent souvent le plaisir de travailler dans la bonne humeur comme élément de satisfaction.

L'atmosphère même étant modifiée, l'impact de la mixité évolue vers une performance sociétale: "du coup, il y a vraiment une ouverture qui se crée en fait chez tout le monde ». Les ressentis des parties prenantes confirment l'idée d'une meilleure atmosphère dans les relations, un respect mutuel. Par ailleurs, la performance sociétale croise la performance individuelle à travers la vision de sa propre performance de la part du dirigeant d'une entreprise. " Arriver à pérenniser une entreprise, je crois que ça, c'est une performance, pérenniser une entreprise et la stabiliser. "

Ces différents niveaux de performance sont interconnectés. Les éléments de performance individuelle, souci du travail bien fait, attention portée à la satisfaction de la clientèle, retentissent sur la performance organisationnelle, estimée tant au niveau de l'entreprise artisanale, qu'à celui de l'organisation que constitue un chantier regroupant plusieurs corps de métiers du bâtiment. La performance organisationnelle, à son tour, 
influence la performance économique, quand bien même, comme mentionné plus haut, l'aspect de rentabilité financière n'apparaît pas directement. Enfin, performance économique et performance sociale sont étroitement imbriquées. Pour tous, la recherche, sinon de l'excellence, du moins du fait de bien faire son métier, concourt à renforcer le sentiment d'appartenance à un monde particulier, celui de l'artisanat. Les valeurs de l'artisan, entre tradition et modernité, articulant savoir-faire et sa transmission d'une part, esprit innovant et entrepreneurial d'autre part, contribuent à élaborer une performance sociétale spécifique. Dans cette évolution sociétale de l'artisanat du bâtiment, la mixité femmes-hommes occupe une place déterminante.

À partir de ces observations, il s'agit donc de percevoir comment ces évolutions peuvent prendre place et être facilitées.

\section{UNE SITUATION EN DYNAMIQUE CONSTANTE}

Conscients du fait que les dirigeants ont une place déterminante dans le changement, il sera utile de faire un détour par leur influence sur l'existence et l'évolution de la mixité, au-delà de ce qui a déjà été traité. Ensuite, un panorama des principales préconisations pourra être proposé.

\section{, 3.1 L'impact des représentations des dirigeants : observer les femmes dirigeantes}

La forte centralisation induit que les dirigeants sont les principaux acteurs de la gestion et du changement. Le constat vaut dans le domaine de l'amélioration de la mixité dans les équipes. L'évolution des représentations des dirigeants constitue donc un élément majeur de l'évolution. Cela n'est pas toujours facile, d'autant que l'entrepreneuriat est né plutôt masculin (De Bruin et al., 2006). Les règles de management sont donc empreintes de codes masculins et traditionnellement, les valeurs masculines comme la compétition et les modes de fonctionnement masculins (réunions tardives, etc.) sont davantage présentes dans les entreprises. Si progressivement les comportements changent, avec des hommes qui recherchent davantage un équilibre de vie, l'enjeu de cette quête reste plus marqué pour les femmes (Naschberger et al., 2013).

Progressivement, la part des femmes entrepreneures s'accroît et les recherches à leur propos se multiplient (Santoni et Barth, 2014). Et ce qui nous intéresse ici est de constater dans quelle mesure les conditions d'exercice entrepreneurial sont différenciées et surtout si leur mode de management varie concernant la mixité et l'intégration de femmes dans l'entreprise, d'autant que certaines études, comme par exemple celle de Carrier et al. (2006), permettent de distinguer des différences.

En ce qui concerne l'intégration des femmes dans l'entreprise, la présence ou l'arrivée d'une femme entrepreneure modifient l'approche de la mixité. II semblerait de plus (Lebègue, 2015) que les femmes aient une vision différente de ce que représente la 
réussite professionnelle, mobilisant des critères moins normatifs et plus subjectifs. Ainsi, l'auteure remet en cause les résultats constatés de la moindre réussite des entrepreneures, supposant l'existence d'une norme de réussite entrepreneuriale universelle. Ces éléments conduisent donc à identifier des différences d'exercice du métier.

Selon Igalens et al. (2011), la femme dirigeante influence de manière évidente sa vision de la mixité dans l'entreprise. Elle va de manière plus naturelle avoir du plaisir à travailler avec des femmes et à les voir évoluer dans leur carrière. On peut même imaginer que le sentiment d'utilité des femmes dirigeantes dans un monde d'hommes soit renforcé. Les entreprises dirigées par des femmes seraient aussi plus respectueuses des équilibres.

Lors d'une table ronde, une entrepreneure artisan a relevé la nécessité de se battre au quotidien pour ne pas laisser intervenir de relations de séduction qui viennent parasiter les échanges professionnels - avec les clients ou les partenaires commerciaux. Elle recrute plus volontiers des apprenties féminines, mais avec difficulté. Elle montre aussi que la discrimination peut se situer non pas en interne, mais également dans le cadre inter-entreprises: une certaine méfiance des entreprises partenaires ou des clients peut s'instaurer pour lui proposer des chantiers, alors même que son expertise est reconnue quand on la sollicite parce qu'il y a un problème et que l'on sait qu'elle est compétente pour le résoudre ... Toute l'ambiguïté apparaît ici Et la dirigeante comprend d'autant mieux les discriminations envers les salariés puisqu'elle les vit comme responsable d'entreprise.

Un autre témoignage rend compte de l'arrivée de la fille du dirigeant aux commandes. Face à la difficulté de recruter, la décision a alors été prise d'embaucher du personnel féminin, malgré de nombreux freins en termes d'équipements à doubler, ou de culture masculine très forte, dans un métier pénible (pollution, vêtements contraignants, etc.). Les deux facteurs déterminants ont clairement été l'arrivée d'une femme dirigeante et le besoin de personnel. Cet exemple est particulièrement utile pour montrer à quel point le changement de représentations peut rendre possible une situation perçue comme inextricable.

Pourtant, il faut rester très prudent, à l'instar de la méta-analyse de Sarah Saint-Michel (2010) qui affirme l'inexistence de différences de compétences de leadership entre hommes et femmes. Elle pointe également l'importance des représentations des différents acteurs (leaders et collaborateurs) qui interagissent en fonction de stéréotypes (les femmes renverraient l'image que l'on attend d'elles). Et le poids des stéréotypes masculins est parfois perpétué par les femmes elles-mêmes (Lambert et RamboarisonLalao, 2008).

Au-delà de ces déterminismes forts, qui ne sont pas immuables, certaines sources de changement peuvent être identifiées. 


\section{, 3.2 Les mesures à mettre en œuvre pour faire évoluer les représentations}

Les mesures à mettre en œuvre pour faire évoluer les représentations - et en particulier celles des dirigeants - ne se cantonnent pas à l'entreprise. Elles concernent également une volonté de la société dans son ensemble.

\subsubsection{Des évolutions sociétales nécessaires}

De manière générale, l'évolution de représentations dans la société passe par de la communication grand public, la diffusion de bonnes pratiques, les retours sur expérience, les travaux de chercheurs, etc. La sensibilisation pour favoriser la mixité au sein de l'artisanat du bâtiment se situe à deux niveaux : rendre le métier attractif en général et le rendre accueillant pour les femmes.

L'attractivité du métier peut être valorisée par ses points positifs comme l'exercice de son métier dans un secteur porteur, non délocalisable, le travail de matériaux nobles pour réaliser du concret et de l'utile, I'utilisation de techniques traditionnelles et modernes, la participation à la préservation/amélioration de l'environnement, l'autonomie, la prise de responsabilités, la gestion de difficultés techniques, la diversité des chantiers ...

Accueillir des femmes dans le métier passe par la reconnaissance et la valorisation des différences, par l'intégration et l'adaptation du secteur, mais surtout par la modification des systèmes de formation existants. Les freins à la mixité dans les métiers du bâtiment seraient ainsi pour partie liés à une représentation négative de ces activités, à la fois dans les familles, et dans certaines branches de l'éducation. II reste donc un gros travail à effectuer non seulement au niveau des parents et des équipes pédagogiques, mais également pour ce qui est de l'autocensure (Carlier et Marlier, 2016). Néanmoins, il semblerait que la prise en compte du degré de masculinité ou de féminité d'un métier relève plutôt d'une question générationnelle (Gavoille et al., 2014) et que les nouvelles générations accordent moins d'importance aux questions de métiers genrés. Notons que la formation et la sensibilisation concernent à la fois les employés et les futurs dirigeants, fait essentiel pour parvenir à une évolution des représentations.

La formation est reconnue comme un levier puissant d'évolution des représentations (Bruna et Chauvet, 2010 ; Klinkenberg, 2016 ; Paradas et al., 2017). Elle apparaît au cœur des procédures à développer et à renforcer pour élargir la mixité. II convient de distinguer entre formation initiale et formation continue.

En formation permanente, le tableau est beaucoup plus favorable, dans la mesure où les femmes y accédant - souvent à la suite d'une reconversion choisie - ne rencontrent pas de difficultés spécifiques.

En formation initiale, intégrer les préoccupations de mixité et combattre les stéréotypes peut se faire dès le plus jeune âge. Les expériences réalisées, par exemple pour favoriser l'accès des femmes aux métiers scientifiques, donnent des résultats probants. 
Concernant l'artisanat du bâtiment, deux voies sont ouvertes: Iycées techniques et apprentissage. II serait souhaitable que les politiques de sensibilisation soient diffusées dans ces filières professionnelles. Cela passera nécessairement par une réforme de l'architecture de l'offre d'enseignement et de formation. En effet, la sous-représentation des filles dans l'apprentissage (20\% entrent en apprentissage, contre $34 \%$ de garçons après un second cycle professionnel) pénalise leur insertion. Ainsi, Naves et WisniaWeill (2014) prévoient qu'il serait possible de générer sur 5 ans, grâce à une politique de mixité ciblée sur des secteurs non-mixtes porteurs, stratégiques, entre 150000 et 180000 apprenties supplémentaires. L'orientation vers les formations techniques est souvent faite par défaut : la plupart des jeunes garçons ne choisissent pas véritablement les métiers du bâtiment. Ils y sont orientés " faute de mieux ". D'où une atmosphère assez difficile dans les classes, et les jeunes filles qui, elles, s'y trouvent à la suite d'un choix personnel, sont souvent découragées par cet environnement. Cette situation est d'autant plus regrettable que des systèmes d'information et d'incitation sont initiés par le rectorat pour promouvoir l'égalité filles-garçons dans les métiers perçus comme " masculins ", et tout particulièrement le secteur du bâtiment.

Les actions à initier dans le domaine de l'éducation exigent de faire évoluer les représentations vis-à-vis des métiers du bâtiment. On connaît la difficulté de modifier les images. Selon nous, deux directions pourraient être envisagées : d'une part, valoriser les métiers du bâtiment, à la fois aux yeux des jeunes gens et des enseignants ; d'autre part, "matraquer" le message, tant à destination des garçons que des filles, selon lequel le secteur du bâtiment n'est pas " réservé » aux hommes. C'est une lutte contre les stéréotypes qu'il faut poursuivre, dépassant très largement le seul champ éducatif. Les instituts de formation initiale ou permanente ne sont pas les seules voies d'accès au changement. La famille, l'école, les pairs, les réseaux professionnels et Pôle Emploi peuvent également être des vecteurs possibles pour attirer plus de femmes dans le métier et modifier les représentations de tous. Des initiatives intéressantes de marrainage, comme celle initiée au lycée Alfred Sauvy de Villelongue dels Monts en $2015^{2}$ montrent leur efficacité pour permettre à la femme de gagner en crédibilité et légitimité.

\subsubsection{Mesures au sein de l'entreprise}

Combattre les stéréotypes : le volet négatif des stéréotypes repose sur une double vision : d'une part, un sentiment de la supériorité masculine dans les métiers et dans les tâches, d'autre part un rabaissement des capacités physiques et des compétences professionnelles des femmes. Ces ressentis sont indéniables. Les stéréotypes restent d'actualité. II convient toutefois de moduler ces impressions négatives. Les entretiens,

[2] https://www.lyc-sauvy-villelonguedelsmonts.ac-montpellier.fr/le-batiment-au-feminin-avec-des-marraines-depromotion-pour-nos-jeunes-diplome-es 
en particulier avec les parties prenantes, font apparaître une vraie volonté de lutter contre les stéréotypes. Le chemin est encore long pour accéder à l'égalité des chances professionnelles, mais les acteurs soulignent une amélioration des représentations et des comportements à travers le temps. Carlier et Marlier (2016) témoignent à propos d'outils visant la déconstruction des stéréotypes genrés dont sont victimes les femmes du secteur de la construction, citant l'action de coaching sectoriel, Femmes et Construction, qui a très tôt développé des outils de sensibilisation et proposé un accompagnement personnalisé. Rencontres, recueil de données, production d'outils, utilisation des médias et des réseaux sociaux, réseaux professionnels... toutes ces démarches montrent leur utilité quant à l'insertion.

Positiver les différences : les femmes engagées dans les activités du bâtiment sont créditées par leurs collègues masculins de capacités relationnelles et organisationnelles supérieures. Si les compétences professionnelles des femmes sont questionnées dans un premier temps - d'où la nécessité de "faire ses preuves"-, elles sont par la suite reconnues. Loin de les nier, il peut être très efficace de mettre l'accent sur ces différences, en insistant par là même sur des éléments de complémentarité, qui aboutiront à une amélioration des résultats (Carlier et Marlier, 2016). Les femmes choisissent d'entrer dans les métiers du bâtiment : leur volonté de pénétrer des métiers a priori masculins, leur vision différenciée des relations de travail, constituent autant d'éléments positifs pour le secteur tout entier, qu'il convient donc de cultiver.

Souligner les synergies : un intérêt de l'étude réside dans la mise en lumière du fait que la mixité femmes-hommes dans les équipes sur les chantiers, dans les collaborations professionnelles, impacte positivement l'efficience et l'efficacité du secteur dans son ensemble. Les conclusions relatives aux différentes dimensions de la performance soutiennent cette assertion. La mixité entraîne un dépassement des performances individuelles. Les résultats peuvent s'apprécier d'un point de vue matériel : satisfaction des clients, finitions soignées, etc. Dans la même perspective, la confrontation femmeshommes des ressentis quant aux conditions de travail, vis-à-vis du traitement d'un problème technique ou commercial, détermine une co-construction dans la résolution d'un problème, et par là même, une élévation du niveau de qualité de la solution apportée. Enfin, l'impact de la mixité qui paraît le plus significatif réside dans une amélioration de l'atmosphère au travail, dans les relations entre professionnels.

La stratégie du pied dans la porte, enfin, montre régulièrement toute son efficacité pour développer des pratiques vertueuses au sein de l'entreprise, par le biais de la sensibilisation du dirigeant. De nombreux travaux sur le développement de la RSE ou de la diversité dans les petites entreprises montrent à quel point c'est le premier pas qui compte (Berger-Douce, 2014 ; Bon, 2014 ; Paradas, 2010). II semble ainsi nécessaire de faciliter et favoriser les premières expériences au sein des entreprises. Le processus mis en œuvre renvoie à la réflexivité qui illustre le renforcement progressif des pratiques et 
la dynamique circulaire permanente des valeurs et représentations. Dans les modèles d'apprentissage (Weil-Barais, 2001) la réflexivité traduit la capacité à pouvoir faire des liens implicites entre les résultats obtenus et leur conduite. Cette réflexivité permet de donner un caractère "renforçant " aux comportements eux-mêmes. Les moyens de favoriser cette réflexivité, dans le cadre de l'accès des femmes au secteur, sont encore à inventer, mais se révéleront sans nul doute très puissants. Une voie de lutte contre les attitudes stéréotypées pourrait passer par les rencontres entre acteurs - à titre d'exemple entre chefs d'entreprises et jeunes filles. Les rencontres sont des mises en situation, et plus elles auront lieu, plus cela obligera de fait à faire évoluer les mentalités. Mais selon certains interlocuteurs, la route est encore longue: On est sur la mixité de genre, ce qu'on y met derrière, qui se confronte aux personnes qui ne souhaitent pas que cela évolue.

Ces propositions sont en phase avec les travaux de Santoni (2018), constatant quatre grands besoins spécifiques d'accompagnement des entrepreneures : cognitifs (être formée), décisionnels (échange et réseau), affectifs (être entourée et guidée) et motivationnels (être encouragée). L'auteure insiste finalement sur l'importance des réseaux et des interactions, tout comme Carlier et Marlier (2016), dans leur article très proche de nos problématiques.

\section{CONCLUSION}

Les stéréotypes positifs et négatifs s'articulent finalement entre trois dimensions : la reconnaissance de différences, l'acceptation de ces différences et la question des compétences.

Trois facteurs témoignent de l'ouverture de l'artisanat du bâtiment vers la mixité femmehomme : le volet positif des stéréotypes, l'entrepreneuriat féminin, et l'appréciation d'une performance. L'aspect positif du stéréotype touchant à la compétence réside dans un comportement d'acceptation, voire d'admiration de la part des collègues masculins, une fois la compétence reconnue (mais la femme doit en avoir fait la preuve, et souvent, démontrer une maîtrise du domaine supérieure...).

Concernant l'esprit d'entreprise des femmes, il est apparu que les femmes de l'artisanat du bâtiment choisissent vraiment leur profession, ce qui rejoint le courant Boundaryless décrit par Lebègue (2015) à propos des approches contemporaines de carrière. II ne s'agit en aucun cas d'un choix par défaut - d'autant plus qu'il leur faut souvent imposer ce choix à l'entourage familial, éducatif et par la suite professionnel. De plus, la difficulté d'entrer dans le métier choisi en tant que salariée conduit souvent les femmes à créer leur propre entreprise. La capacité professionnelle est alors croisée avec la capabilité entrepreneuriale.

Enfin, la question de la relation mixité-performance constitue un élément clé de l'étude. Il convient de souligner le caractère systémique de la relation mixité-performance. 
On constate une interaction entre les différentes dimensions de la performance, fondée sur des boucles de retour et de relance. La présence de femmes à l'intérieur de l'entreprise, ou en tant que collègues, se traduit par une amélioration de l'ensemble des comportements professionnels. Les dirigeants ne peuvent qu'être sensibles à cette dimension, qui peut les conduire à faire évoluer leurs représentations.

Finalement, du point de vue de l'estimation de la mixité femme-homme dans les entreprises artisanales du bâtiment, la balance entre aspects défavorables, tels les stéréotypes négatifs, les freins à la mixité, et aspects favorables, volet positif des stéréotypes, motivations entrepreneuriales, contribue à faire apparaître un état de la mixité en progression, mais encore à largement soutenir et renforcer. Toutefois, l'évolution constatée à travers le temps permet de dégager une perspective plutôt favorable. Au total, nos résultats montrent des changements profonds de perception et de comportement. Les femmes s'engagent dans ce secteur et leur professionnalisme semble de plus en plus reconnu. Leur présence dans les métiers modifie les codes, de sorte que la mixité est perçue comme un facteur d'amélioration de la performance globale.

L'étude qui a été réalisée peut évidemment comporter des limites concernant les échantillons et la méthode. D'autres régions pourraient être explorées afin d'identifier d'éventuelles différences. Si les entretiens individuels ou par groupes semblent mieux adaptés que des études quantitatives pour l'approche des représentations, d'autres méthodes, plus proches de la psychosociologie, pourraient être exploitées. De plus, dans une étude qualitative, il est toujours possible d'avoir des biais de représentativité, même si dans le cas présent, les approches ont été volontairement très larges. Plusieurs chercheurs ont été à l'œuvre pour cette étude, ce qui permet de réduire sensiblement les problèmes de subjectivité.

Enfin, ces constats peuvent s'inscrire dans le spectre plus général de la prise en compte de la RSE dans les petites entreprises. La mixité, et tout particulièrement la mixité femmes-hommes, constitue une composante de la responsabilité sociale des entreprises, y compris des plus petites, y compris des entreprises artisanales du bâtiment. Le jeu des synergies est ici encore à convoquer : il est assez remarquable de constater que les informations ou formations touchant à la RSE sont le plus souvent suivies par des femmes dirigeantes ou salariées d'entreprises artisanales. Les valeurs relatives à la RSE rejoignent d'ailleurs les valeurs dites "féminines ", mais également les valeurs fondamentales du monde de l'artisanat. Une sensibilisation à la RSE, une meilleure connaissance de ses contenus, viendrait à terme renforcer la mixité femmes-hommes dans l'artisanat du bâtiment, tout comme la mixité vient étayer l'engagement socialement responsable des dirigeants. 


\section{RÉFÉRENCES}

ABRIC, J.C. (2011). Pratiques sociales et représentations. Presses Universitaires de France.

ACHIN, C., MEDA, D. \& WIERINK, M. (2005). Mixité professionnelle et performance des entreprises, le levier de l'égalité. Rapport DARES 91, janvier.

BELGHITI-MAHUT, S., LAFONT, L., RODHAIN, A., \& RODHAIN, F. (2014). Quand les femmes au sommet se racontent. @GRH, 2014/1, n 10, p. 59-85.

BELGHITI, S. \& RODHAIN, F. (2001). Les femmes constituent-elles un potentiel pour les entreprises? La Revue des Sciences de Gestion : Direction et Gestion, Juil-0ct 2001, p. $190-205$.

BENDER, A.F. (2004). Egalité professionnelle ou gestion de la diversité - Quels enjeux pour l'égalité des chances? Revue Française de Gestion, 4 (151), p. 205-217.

BENDER, A.F. \& PIGEYRE, F. (2003). L'égalité professionnelle entre hommes et femmes comme responsabilité sociale de l'entreprise. IAP, p. 78-86.

BERGER-DOUCE, S. (2014). La gestion par la RSE, un gage de performance en PME? In : Louart, P. \& Vilette, M.A., Gérer les RH dans les PME, Vuibert Recherche, AGRH, p. 355-374.

BON, V. (2014). Les enjeux sociaux de la responsabilité des petites entreprises. In: Louart, P. \& Vilette, M.A., Gérer les RH dans les PME, Vuibert Recherche, AGRH, p. 333-354.

BRUNA, M.G. \& CHAUVET, M. (2010). La diversité, levier de performance... sous condition de management. Cahier de recherche $n^{\circ} 2$, juillet, Chaire Management \& Diversité Dauphine.

CARRIER, C., JULIEN, P.A., \& MENVIELLE, W. (2006). Entrepreneuriat féminin : une synthèse des études des 25 dernières années. Gestion, 31 (2), p. 36-50.

CATALYST (2004). The Bottom Line. Connecting Corporate Performance and Gender Diversity. New York. https://www.catalyst.org/system/files/The_Bottom_Line_ Connecting_Corporate_Performance_and_Gender_Diversity.pdf

CARLIER, A. \& MARLIER, A. (2016). Choix de femme pour métier (dit) d'homme, quand la démarche collective soutient les possibles individuels. Les Cahiers Internationaux de Psychologie Sociale, 110 (2), p. 271-293.

CESE (2014). Agir pour la mixité des métiers. Les avis du conseil économique, social et environnemental. Rapports publics, rapporteur Patrick Liebus.https://www.ladocumentationfrancaise.fr/var/storage/rapports-publics/144000737.pdf 
CHICHA, M.T. (2013). Inégalités de genre et pratiques d'entreprises au Maroc. BIT. Genève.

CNISF (2014). Enquête 2014 des ingénieurs et scientifiques de France. http://pmb.cereq. $\mathrm{fr} /$ doc_num.php?explnum_id=1609

CORNET, A. \& WARLAND, P. (2008). GRH et gestion de la diversité. Dunod.

CORNET, A. \& CONSTANTINIDIS, C. (2004). Entreprendre au féminin : Une réalité multiple et des attentes différenciées. Revue française de Gestion, 151(4), p. 191-204.

CORNET, A. \& BONNIVERT, S. (2008). Leadership et genre. In : Cornet, A., Laufer, J. \& Belghiti, S., Genre et GRH, Les défis de l'égalité hommes-femmes, Vuibert, p. 125-138.

COURNUT-JANIN, M. (2000). Féminin et Féminité. $2^{2}$ édition. PUF.

COUSIN, 0. (2007). La construction des inégalités homme/femmes dans l'entreprise. Une analyse de cas dans la métallurgie. Sociologie du travail, 49(2), p. 195-219.

DAUNE-RICHARD, A.M. (1998). Qualification et représentations sociales. In : Femmes, genre et société, l'état des savoirs, La Découverte.

DE BRUIN, A., BRUSH, C.G. \& WELTER, F. (2006). Introduction to the special issue: towards building cumulative knowledge on women's entrepreneurship. Entrepreneurship Theory \& Practice, 30 (5), p. 585-593.

DUBAR, C. (1998). Trajectoires sociales et formes identitaires. Clarifications conceptuelles et méthodologiques. In Sociétés Contemporaines, 29, p. 73-85.

DUPUIS, J.-C., HANED, N. \& LE BAS, C. (2006). La RSE en Rhône-Alpes. Premiers résultats d'une enquête auprès des PME régionales. Bulletin EFconomia Humana, Édition spéciale sur la RSE et les PME, ESG, UQAM, 4(11), décembre, p. 9-12.

FALCOZ, C. (2016). Égalité, discrimination, diversité \& GRH. Une thématique transversale enrichissant la recherche en comportement organisationnel. Revue internationale de psychosociologie et de gestion des comportements organisationnels, 2016/54 (Vol. XXII), p. 139-152.

GALLIOZ, S. (2006). Force physique et féminisation des métiers du bâtiment. Travail, genre et sociétés, 16(2), p. 97-114.

GAUZENTE, C. (2000). Mesurer la performance des entreprises en l'absence d'indicateurs objectifs: quelle validité ? Analyse de la pertinence de certains indicateurs. Finance Contrôle Stratégie, 3(2), p. 145-165.

GAVOILLE, F., LEBEGUE, T. \& PARNAUDEAU, M. (2014). Le métier a-t-il toujours un genre? Une question de génération. I.M.C.F, Question(s) de management, 2(6), p. 111-123.

GOY, H. (2006). Quel statut pour les PME dans la recherche francophone et stratégie? XV' Conférence internationale de Management stratégique, Annecy / Genève, juin. 
IGALENS, J., LOUITRI, A., \& SAHRAOUI, D. (2011). GRH et encadrement au féminin : cas d'une PME marocaine. Management \& Avenir, 3(43), p. 82-102.

JODELET, D. (2003). Les représentations sociales. PUF, coll. Sociologie d'aujourd'hui, 7 édition.

KLINKENBERG, M. (2016). Le mauvais genre? Genre, sexe et société. Les Cahiers Internationaux de Psychologie sociale, PUL, 2016/2, n 110, p. 247-269.

LAMBERT, G., \& RAMBOARISONLALAO, L. (2008). Genre et diversité : un regard sur les pratiques de GRH dans la région Alsace. 20 Congrès annuel de I'AGRH, Dakar, 9-12 novembre.

LANDRIEUX-KARTOCHIAN, S. (2005). L'intérêt managérial des démarches d'égalité professionnelles, un exemple de (ré)conciliation de l'économique et du social ? $16^{e}$ congrès annuel de I'AGRH, Paris Dauphine, 15-16 septembre.

LAUFER, J. (2003). L'accès des femmes à la sphère de direction des entreprises: la construction du plafond de verre. DARES, rapport de synthèse, octobre.

LEBÈGUE, T. (2015). La réussite de la carrière entrepreneuriale des femmes. Revue de l'entrepreneuriat, 2015/1, vol 14, p. 93-127.

LEYENS, J.-P., YZERBYT, V., \& SCHADRON, G. (1996). Stéréotypes et cognition sociale. Mardaga.

LOUEY, S., \& SCHUTZ, G. (2014). Les effets de la mixité au prisme du corps et de la sexualité, Travail et Emploi, 140, p. 5-19.

LUYCKX, M. (2002). Le rôle de l'expert: participer au ré-enchantement du monde. Reflets et perspectives de la vie économique, XLI, 0(1), p. 89-99.

MAHÉ DE BOISLANDELLE, H. (1996). Effet de grossissement et management des ressources humaines en PME. $3^{e}$ Congrès International Francophone sur la PME, Trois Rivières, Québec.

MARSAN, C. (2007). Au-delà du masculin et du féminin. Cahiers de psychologie politique, 11, http://lodel.irevues.inist.fr/cahierspsychologiepolitique/index.php?id=581, 2007.

MEYNAUD, H.Y., FORTINO, S. \& CALDERON, J. (2009). La mixité au service de la performance économique: réflexions pour penser la résistance. Cahiers du Genre, 47, p. 15-33.

MOSCIBICI, S. (1961). La psychanalyse, son image et son public. PUF.

NASCHBERGER, C., QUENTAL, C., \& LEGRAND, C. (2013). Étapes de carrière et perception des enjeux en matière d'équilibre vie professionnelle - vie personnelle chez les cadres hommes et femmes. 1ère Journée du Management Socialement Responsable des Entreprises, Mixité et diversité des équipes, France Business School. 
NAVES, M.C. \& WISNIA-WEILL, V. (2014). Les stéréotypes filles-garçons, terreau des inégalités hommes-femmes et enjeu de justice sociale? In : Naves, M-C. \& WisniaWeill V., Lutter contre les stéréotypes filles-garçons - un enjeu d'égalité et de mixité dès l'enfance. Commissariat général à la stratégie et à la prospective, Rapports et Documents, janvier, p. 21-27.

PARADAS, A. (2007). Le dirigeant comme levier de la RSE en TPE. Approche exploratoire basée sur l'utilisation de récits et d'une cartographie cognitive. Revue Internationale PME, Numéro spécial : Les PME et la responsabilité sociale des entreprises, 20 (3-4), p. 43-67.

PARADAS, A. (2008). Proximité représentative et responsabilité sociale dans les TPE. In : Torres, 0. \& Jaouen, A., Les Très Petites Entreprises : un management de proximité, L'Hermès Lavoisier, p. 125-141.

PARADAS, A. (2010). Les aspects humains de la RSE en petite entreprise. In : Louart, P. \& Vilette, M.A., La GRH en PME, Vuibert Recherche, AGRH, p. 377-387.

PARAdAS, A., REVELLI, C., DEBRAY, C., COURRENT, J-M. \& SPENCE, M. (2017). Pratiques responsables des dirigeants de PME : influence du profil du dirigeant. Revue de l'Entrepreneuriat, 16(3-4), p. 197-228.

PIERRE, P., MUTABAZI, E. \& SADIK, Y. (2008). Promouvoir en entreprise des politiques de gestion de la diversité ? Perspectives comparées franco-marocaines. Actes du Congrès de l'AGRH, Dakar.

POLGE, M., FOURCADE, C., DEBRAY, C. \& PARADAS, A. (2017). Femmes dans l'entreprise, Questions de société. EMS Management et Société.

SAINT-MICHEL, S. (2010). L'impact du genre sur le style de leadership : I'importance d'introduire les traits de personnalité des leaders. Revue Internationale de Psychosociologie, 40, p. 181-203.

SANTONI, J. (2018). Quels processus pour répondre aux besoins spécifiques des entrepreneures? Entreprendre \& Innover, 2018/1 (n 36), p. 29-40.

SANTONI, J. \& BARTH, I. (2014). Le rôle du réseau dans le développement de l'entrepreneuriat féminin : cas d'un centre entrepreneurial au sein d'une business school. @GRH, 2014/2, no 11, p. 81-113.

SCOTTO, M.J., SAPPE, R. \& BOYER, A. (2008). Réussir la diversité du genre. Une expérience de développement de l'égalité professionnelle femme/homme dans le secteur de la construction, souvent considéré comme " masculin » : l'exemple de CARI, entreprise de BTP dans les Alpes Maritimes. Management \& Avenir, 18(4), p. 18-41.

SENAC, R. (2015). L'égalité sous conditions. Les Presses Sciences-Po Paris.

SAINT PIERRE, J., CARRIER, C., \& PILAEVA, K. (2011). Développement durable et PME : les femmes ont-elles une conception différente de celle des hommes? Colloque 
International: PME en marche vers le développement durable, Montréal, Octobre. $<$ hal-01703402>.

TORRES, 0. (2004). The SME concept of Pierre-André Julien: An analysis in terms of proximity. Piccola impresa, Small Business, 2, p. 1-12.

TOSTAIN, M. (2016). Pour en finir avec la domination masculine ? Regard critique sur les études psychosociales des relations entre sexes. Bulletin de Psychologie, 2016/5, $n^{\circ} 545$, p. 345-363.

VIDAL, C. (dir.) (2006). Féminin/masculin : mythes scientifiques et idéologie. Belin.

VIER MACHADO, H., \& ROULEAU, L. (2002). Identité sociale et entrepreneuriat féminin : étude comparative entre le Brésil et le Canada. $6^{e}$ Congrès international francophone sur la PME, HEC - Montréal, octobre.

WEIL-BARAIS, A. (dir.) (2001). L'homme cognitif. PUF, 6e Edition.

ZUINEN, N., (2002). Essai sur le rôle des femmes et des valeurs féminines. Reflets et perspectives de la vie économique, 1(XLI), de Boeck Université, p. 109-114.

\section{ANNEXE 1. PRÉSENTATION DES ACTEURS DE L'ÉTUDE}

\section{, 1.1 Caractéristiques des artisan.e.s}

\begin{tabular}{|l|c|c|}
\hline Activité & Taille de l'entreprise - sexe & Age \\
\hline Architecture d'intérieur - Coloriste & 0 salarié - Dirigeante & $\mathbf{4 5}$ ans \\
\hline $\begin{array}{l}\text { Architecture d'intérieur - } \\
\text { Coordination Travaux }\end{array}$ & 0 salarié - Dirigeante & $\mathbf{5 7}$ ans \\
\hline Cuisiniste & 0 salarié - Dirigeante & $\mathbf{4 5}$ ans \\
\hline Construction maisons ossature bois & $<10-$ Dirigeant & $?$ \\
\hline Menuiserie & 9 salariés - 2 dirigeants (H/F) & $\mathbf{4 8}$ ans \\
\hline $\begin{array}{l}\text { Menuiserie - Charpente - } \\
\text { Rideaux Fer Inox }\end{array}$ & 8 salariés - 2 dirigeants (H/H) & $\mathbf{5 7}$ ans \\
\hline Métallerie - Chaudronnerie & 0 salarié - Dirigeante & $\mathbf{3 4}$ ans \\
\hline Peinture & 0 salarié - Dirigeante & $\mathbf{6 4}$ ans \\
\hline Peinture - Faïencerie murale & 0 salarié - Dirigeante & $\mathbf{4 5}$ ans \\
\hline Plomberie - Chauffage & 0 salarié - 2 dirigeants (H/F) & $\mathbf{3 0}$ ans \\
\hline Plomberie - Chauffage & 60 salariés - Dirigeant & $\mathbf{5 3}$ ans \\
\hline $\begin{array}{l}\text { Plomberie - Installation Salles } \\
\text { de bains }\end{array}$ & 0 salarié - Dirigeante & $\mathbf{3 0}$ ans \\
\hline Travaux accès difficiles (cordistes) & $>10-2$ dirigeants (H/F) & $\boldsymbol{?}$ \\
\hline
\end{tabular}


- 1.2 Caractéristiques des parties prenantes (* certaines données d'âge n'ont pas été renseignées)

\begin{tabular}{|c|c|c|c|}
\hline Nom organisme & Activité & \begin{tabular}{|c|} 
Statut de \\
I'interlocuteur - tranche d'âge
\end{tabular} & Genre \\
\hline AFPA FEMME & $\begin{array}{c}\text { Formation professionnelle, } \\
\text { domaine bâtiment }\end{array}$ & Direction $-45 / 50$ ans & $\mathbf{F}$ \\
\hline $\begin{array}{l}\text { Atout Métiers } \\
\text { Carif-Oref }\end{array}$ & $\begin{array}{l}\text { Observation et information } \\
\text { sur formation professionnelle }\end{array}$ & Responsable département* & H \\
\hline CARSAT & $\begin{array}{l}\text { Organisme sécurité sociale } \\
\text { à compétence régionale }\end{array}$ & Ingénieur conseil sécurité ${ }^{*}$ & H \\
\hline CFA Bâtiment & $\begin{array}{c}\text { Formation professionnelle } \\
\text { par apprentissage }\end{array}$ & $\begin{array}{c}\text { Formation - En charge relations } \\
\text { Entreprises }-35-45 \text { ans }\end{array}$ & $\mathbf{F}$ \\
\hline CDIFF & $\begin{array}{l}\text { Information sur le droit } \\
\text { des femmes }\end{array}$ & $\begin{array}{c}\text { Conseil en insertion } \\
\text { professionnelle* }\end{array}$ & $\mathbf{F}$ \\
\hline $\mathrm{ClO}$ & $\begin{array}{c}\text { Orientation des jeunes } \\
\text { en formation initiale }\end{array}$ & Direction $-45-50$ ans & $\mathbf{F}$ \\
\hline $\mathrm{ClO}$ & $\begin{array}{c}\text { Orientation des jeunes } \\
\text { en formation initiale }\end{array}$ & $\begin{array}{c}\text { Conseil en orientation } \\
\text { Psychologue }-40-50 \text { ans } \\
\end{array}$ & $\mathbf{F}$ \\
\hline GRETA FEMME & $\begin{array}{l}\text { Développement } \\
\text { de la formation }\end{array}$ & Conseil en formation continue* & H \\
\hline Union Matériaux & $\begin{array}{c}\text { Vente matériaux } \\
\text { pour activité second œuvre }\end{array}$ & Direction régionale 35-45 ans & $\mathbf{H}$ \\
\hline
\end{tabular}

\section{ANNEXE 2. PARTICIPANTS CONVOQUÉS AUX FOCUS GROUPES}

Présidentes CAPEB, Présidentes Commission Femmes de l'artisanat du bâtiment, Elues CAPEB, Présidente CDFA, Cheffe d'entreprise, Peintre en bâtiment (Entrepreneuse salariée SCOP) ; Conjointe associée, Conjointe salariée, Gérante, Secrétaire Général et chargée de mission CAPEB, Secrétaires CAPEB dont une est conjointe dans une entreprise de Maçonnerie, Conjointe clim-chauffage, Conjointe couvreur, Université, Force Femmes, Entreprendre au féminin, DIRECCTE, FCE, Dirigeante d'une entreprise Réseau LCE, Bâtir au Féminin, CFE CGC, CFTC, Directeur du CFA-BTP, Députée, FACE, Vice-président collectivité, Maire-adjoint, Directrice déléguée Région, Union régionale CIDFF : Vice-présidente Commission égalité Femmes-Hommes, Avocats, Dirigeantes d'entreprises, Expert-comptable, artisan maçon élu CAPEB, Inspecteur Education Nationale (information et orientation), Développeur de l'apprentissage CFA, Artisan messager (Menuiserie), Directeur SEGPA collège, Professeur en SEGPA, Collectif $I A E$, Al Passerelles, UR missions locales, CD - IAE/ESS, chargée de mission CABEB, OPPBTP : responsable agence, Cabinet de conseil en développement : dirigeante, GEIO BTP, ECOBATP, DG Medef, U2P, Cabinet recrutement. 